Behavioral/Cognitive

\title{
Specifying a Causal Role for Angular Gyrus in Autobiographical Memory
}

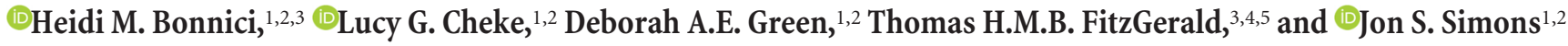 \\ ${ }^{1}$ Department of Psychology, University of Cambridge, Cambridge, United Kingdom CB2 3EB, ${ }^{2}$ Behavioural and Clinical Neuroscience Institute, University \\ of Cambridge, Cambridge, United Kingdom CB2 3EB, ${ }^{3}$ School of Psychology, University of East Anglia, Norwich, United Kingdom NR4 7TJ, ${ }^{4}$ Wellcome \\ Trust Centre for Neuroimaging, University College London, London, United Kingdom WC1N 3AR, and ${ }^{5}$ Max Planck-UCL Centre for Computational \\ Psychiatry and Ageing Research, London, United Kingdom WC1B 5EH
}

Considerable recent evidence indicates that angular gyrus dysfunction in humans does not result in amnesia, but does impair a number of aspects of episodic memory. Patients with parietal lobe lesions have been reported to exhibit a deficit when freely recalling autobiographical events from their pasts, but can remember details of the events when recall is cued by specific questions. In apparent contradiction, inhibitory brain stimulation targeting angular gyrus in healthy volunteers has been found to have no effect on free recall or cued recall of word pairs. The present study sought to resolve this inconsistency by testing free and cued recall of both autobiographical memories and word-pair memories in the same healthy male and female human participants following continuous theta burst stimulation (cTBS) of angular gyrus and a vertex control location. Angular gyrus cTBS resulted in a selective reduction in the free recall, but not cued recall, of autobiographical memories, whereas free and cued recall of word-pair memories were unaffected. Additionally, participants reported fewer autobiographical episodes as being experienced from a first-person perspective following angular gyrus cTBS. The findings add to a growing body of evidence that a function of angular gyrus within the network of brain regions responsible for episodic recollection is to integrate memory features within an egocentric framework into the kind of first-person perspective representation that enables the subjective experience of remembering events from our personal pasts.

Key words: memory; parietal lobe; recollection; transcranial magnetic stimulation

\section{Significance Statement}

In seeking to understand the role played by the angular gyrus region of parietal cortex in human memory, interpreting the often conflicting findings from neuroimaging and neuropsychology studies has been hampered by differences in anatomical specificity and localization between methods. In the present study, we address these limitations using continuous theta burst stimulation in healthy volunteers to disrupt function of angular gyrus and a vertex control region. With this method, we adjudicate between two competing theories of parietal lobe function, finding evidence that is inconsistent with an attentional role for angular gyrus in memory, supporting instead an account in terms of integrating memory features within an egocentric framework into a firstperson perspective representation that enables the subjective experience of remembering.

\section{Introduction}

Of the network of brain areas associated with episodic memory, one region to receive considerable attention recently is parietal

Received May 15, 2018; revised Aug. 31, 2018; accepted Sept. 14, 2018.

Author contributions: H.M.B. and J.S.S. designed research; H.M.B., L.G.C., and D.A.E.G. performed research; H.M.B., T.H.M.B.F., and J.S.S. analyzed data; H.M.B., L.G.C., D.A.E.G., T.H.M.B.F., and J.S.S. wrote the paper.

This work was supported by a James S. MCDonnell Foundation Scholar Award to J.S.S. It was completed within the University of Cambridge Behavioural and Clinical Neuroscience Institute, funded by a joint award from the United Kingdom Medical Research Council and the Wellcome Trust. We thank Zoe Kourtzi and Andrew Welchman for use of their TMS system.

The authors declare no competing financial interests.

Correspondence should be addressed to Dr. Jon S. Simons, Department of Psychology, University of Cambridge,

Downing Street, Cambridge CB2 3EB, United Kingdom. E-mail: jss30@cam.ac.uk.

https://doi.org/10.1523/JNEUROSCl.1239-18.2018 cortex. Wagner et al. (2005) highlighted the common occurrence of parietal activity in neuroimaging studies of recollection, particularly in the angular gyrus. This frequency might suggest a critical role in memory function. However, highly accurate memory performance is observed even in patients whose lesions overlap closely with the areas activated by healthy participants performing the same memory tasks (Simons et al., 2008). As such, there is much to understand about the role played by parietal cortex in memory abilities.

This is an open-access article distributed under the terms of the Creative Commons Attribution License Creative Commons Attribution 4.0 International, which permits unrestricted use, distribution and reproduction in any medium provided that the original work is properly attributed. 


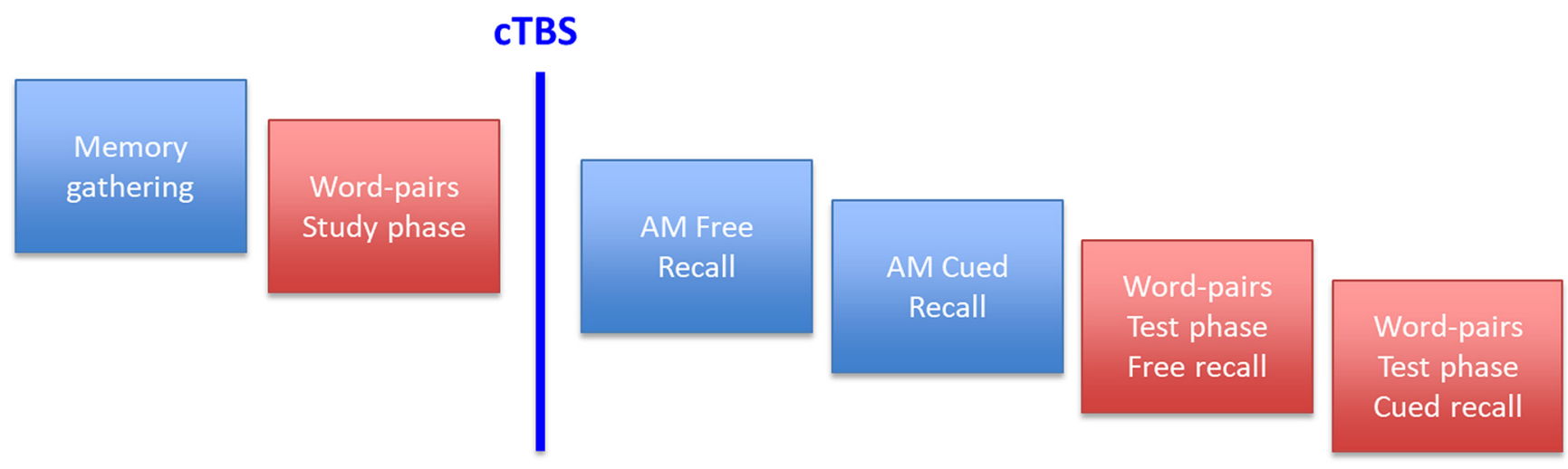

Figure 1. Design of the experiment.

Although accurate memory performance can be observed following parietal lesions, memory is not entirely unaffected. Patients with parietal damage have been reported to exhibit impairment when freely recalling autobiographical events from their personal pasts, despite their memories appearing intact when recall is cued by specific questions about the events (Berryhill et al., 2007). In addition, although accuracy in identifying the context in which stimuli were previously encountered (source memory) tends to be unaffected by parietal lesions, participants' confidence in their accurate recollections can be significantly reduced (Simons et al., 2010). Several theories have been proposed to explain these findings, including that free recall and recollection confidence are impaired following parietal damage because of a reduced tendency for memories to capture attention spontaneously (Cabeza et al., 2008; Ciaramelli et al., 2010a), or that they might reflect a diminished subjective experience of "re-living" personal events (Simons et al., 2010; Moscovitch et al., 2016). Yazar et al. (2014) attempted to distinguish these accounts using continuous theta burst stimulation (cTBS) to disrupt angular gyrus function in healthy volunteers. The authors tested for greater impairment of free recall than cued recall of word pairs, as the attentional account would predict, or greater impairment of source recollection confidence than accuracy, consistent with the subjective experience account. The results indicated that free recall and cued recall were unaffected by stimulation of angular gyrus compared with a vertex control location, but that there was selectively reduced confidence in participants' accurate source recollection responses (Yazar et al., 2014). The findings were interpreted as consistent with the proposal that angular gyrus enables the subjective experience of remembering (see also Yazar et al., 2017).

One issue with this interpretation is that the lack of free recall impairment following angular gyrus cTBS observed by Yazar et al. (2014) appears to contradict the result reported in patients with parietal damage by Berryhill et al. (2007). However, Berryhill et al. (2007) tested free and cued recall of autobiographical memories in neuropsychological patients, whereas Yazar et al. (2014) tested free and cued recall of word pairs in healthy volunteers using neurostimulation. In the present study, we sought to resolve this question by assessing free and cued recall of both autobiographical memories and word-pair memories in the same participants following angular gyrus cTBS. If the attentional account is correct, free recall of both types of memories should be more impaired than cued recall because free recall relies more on memories capturing attention spontaneously (Cabeza et al., 2008). If the subjective experience account is correct, there should be a selective reduction in free recall of autobiographical memories, but not word-pair memories, because autobiographical recall relies more on subjectively reliving personal events (Moscovitch et al., 2016).

We also tested another prediction of the subjective experience account: that angular gyrus enables the first-person reexperiencing of past events by integrating memory features within an egocentric framework. Patients with parietal lesions are impaired on egocentric spatial navigation tasks but not allocentric, map-based spatial tasks that are sensitive to hippocampal damage (Ciaramelli et al., 2010b). It may be, therefore, that angular gyrus is responsible for the ability to remember previous events from an egocentric, rather than allocentric, viewpoint. If this account is correct, angular gyrus cTBS should lead to a reduced tendency for participants to report experiencing autobiographical memories from a first-person perspective.

\section{Materials and Methods}

Participants. Twenty-two healthy, right-handed participants (11 female, 11 male) took part in the study (mean age 23.7 years, $\mathrm{SD}=3.9$ years, range $=19-35$ years). All had normal or corrected-to-normal vision, had normal hearing, and gave written consent to participation in a manner approved by the Cambridge Psychology Research Ethics Committee.

Procedure. All participants were tested on two separate occasions, one week apart, in which one session was the experimental condition (stimulation to the left angular gyrus) and the other session was a control session (stimulation to vertex). Participants were counterbalanced to receive left angular gyrus or vertex stimulation first. For each session, all participants followed the same procedure (Fig. 1): an autobiographical memory (AM) gathering phase, a study phase for the word-pairs task, the cTBS procedure, followed by the autobiographical memory recall phase and the word-pairs test phase. Participants received identical stimulation to the angular gyrus and vertex sites and were blind to the experimental hypotheses. The order of the autobiographical and word-pair memory tasks was counterbalanced across participants to control for any stimulation latency effects. Audio responses were recorded using the software Audacity (http://www.audacityteam.org/).

Autobiographical memory. The method used in this study to retrieve and analyze autobiographical memories was a modified version of the Autobiographical Interview (Levine et al., 2002; Rosenbaum et al., 2004). Participants followed the same procedure for both stimulation sessions. Before stimulation, participants were given 5 min to name five significant events from four life periods: one event from childhood (up to the age of 10 years old), one event from adolescence (11-16 years old), two events from early adulthood (17 years old to before the last year), and one event from the previous year. Different events were elicited for each stimulation session, and the titles of each of these memories were written down by the experimenter. Participants were encouraged to select memories that were clear and vivid to them, rich in detail, and that unfolded in an event-like manner, so that they felt like they were reexperiencing the event in their minds as they remembered it. After stimulation, partici- 
Table 1. Autobiographical memory characteristics ${ }^{a}$

\begin{tabular}{lccrrr}
\hline & & & \multicolumn{2}{c}{ Vertex versus AnG } \\
\cline { 5 - 6 } Variable & Vertex [mean (SD)] & AnG [mean (SD)] & & $t$ & $p$ \\
\hline Vividness & $4.33(0.7)$ & $4.39(0.68)$ & & 0.668 & 0.511 \\
Recall frequency & $2.46(0.93)$ & $2.53(0.64)$ & & 0.448 & 0.659 \\
Personally significant (then) & $4.45(0.79)$ & $4.67(0.76)$ & & 1.164 & 0.257 \\
Personally significant (now) & $2.98(0.72)$ & $3.35(1.05)$ & & 1.742 & 0.096 \\
Free recall time (min) & $1.5(0.39)$ & $1.47(0.32)$ & -0.976 & 0.34 \\
No. of general probes & $2.86(2.55)$ & $2.91(2.72)$ & & 0.934 \\
\hline
\end{tabular}

${ }^{a}$ Ratings were on a scale of 1 to 5 , where 1 is the minimum and 5 is the maximum.

pants underwent a free recall phase and then a cued recall phase for each autobiographical memory, lasting $\sim 20 \mathrm{~min}$ in total. During the free recall phase, they verbally described the event without any interruption until they reached the natural end of the account. If the description was too brief or not very detailed, general probes were used to encourage more information (such as "can you remember anything else?"). After freely recalling the event, participants were asked six specific questions by the experimenter to invoke cued recall of additional autobiographical details that were not spontaneously recalled during the free recall phase. The questions were as follows: When did this event take place? Where did this event take place? Do you have any visual images associated with this memory? Do you have any other sensory details (sounds/smell/taste) associated with this memory? Any physical sensations (texture/pain/ temperature)? Can you tell me anything about what you were thinking or feeling at the time? Participants were also asked whether they experienced the recollection from a first-person or a third-person perspective, and rated each memory along a number of parameters (Table 1).

Each interview was then transcribed and scored according to the Levine et al. (2002) method by two independent raters who were blinded to stimulation condition (inter-rater reliability of $r=0.96$ and intraclass correlation of $r=0.94$ ). Scoring was based on the number and type of details each recollection contained. Internal details (specific details about the event in question) were categorized into five types, namely, event, perceptual, time, location, and emotional (thoughts or feelings). External details (details that had no relevance to the event being remembered) were also categorized across these five categories but also included semantic facts, repetition, and irrelevant utterances.

Word-pair memory task. Stimuli for the word-pair memory task were adapted from the previous study of Yazar et al. (2014). Briefly, two sets of 64 noun pairs were used, one set for each session (counterbalanced). Words were randomly allocated to pairs. During the study phase, before stimulation, participants were presented with each word-pair visually and auditorily using Psychopy (http://www.psychopy.org). Each trial was allocated $10 \mathrm{~s}$, and the participants had up to this amount of time to create a sentence that contained both nouns and say it aloud. The test phase after stimulation consisted of two sections, assessing free recall and cued recall, lasting $\sim 10 \mathrm{~min}$ in total. During free recall, the participants were asked to retrieve as many of the words from the study phase as they could remember in $2 \mathrm{~min}$. Participants said each word aloud and were recorded. During cued recall, the participants were randomly presented with one of the two words from each pair and had $3 \mathrm{~s}$ to recall the other word that completed the pair.

cTBS procedure. The cTBS procedure used in this experiment was the standard conditioning protocol used in previous studies (Huang et al., 2005; Yazar et al., 2014, 2017), using a Rapid ${ }^{2}$ (Magstim) transcranial magnetic stimulation device with a standard 70-mm-diameter figure-ofeight coil. On arrival for the first session, each participant had their resting motor threshold assessed for the right first dorsal interosseous hand muscle. Once the autobiographical memory gathering phase and word-pairs study phase were completed, the participant's head was coregistered to their structural MRI via previously identified anatomical landmarks using the neuro-navigation system software Brainsight (Rogue Research). To guide frameless stereotaxy, we used an angular gyrus center of mass with MNI coordinates $(-43,-66,38)$ obtained from a review of the parietal lobe and memory (Vilberg and Rugg, 2008), and a vertex center of mass with MNI coordinates $(0,-15,74)$ obtained from a probabilistic anatomical atlas (Okamoto et al., 2004). A standard conditioning cTBS protocol was then delivered with three pulses at $50 \mathrm{~Hz}$ repeated every $200 \mathrm{~ms}$ for $40 \mathrm{~s}$ at $70 \%$ of the individual's resting motor threshold, to one of the two target areas.

Experimental design and statistical analysis. The anonymized data are openly available from the University of Cambridge data repository at http://doi.org/10.17863/CAM.26398. To explore whether cTBS affected autobiographical memory, repeated-measures ANOVAs were undertaken with factors that included the number and type (internal or external) of details for free and cued recall following each stimulation condition. Repeated-measures ANOVAs were also used to explore whether cTBS affected word-pair memory, contrasting the number of words successfully retrieved during free and cued recall following each stimulation condition. The variable of interest when examining the subjective perspective during autobiographical memory recall was the mean number of memories reported as being experienced in the first-person rather than a third-person perspective. Due to experimenter error, data on perspective were not obtained for 3 of the participants, so analysis was performed on the remaining 19 participants and a paired $t$ test used. A threshold of $p<0.05$ was used throughout.

Effect sizes were calculated using Cohen's $d$ or partial $\eta$-squared $\left(\eta_{\mathrm{p}}^{2}\right)$, as appropriate. For any nonsignificant results observed, Bayes factors (BFs) were computed using JASP software (http://jasp-stats.org/) to establish the strength of evidence for the null hypothesis (Dienes, 2014). BFs of $>3$ were interpreted as substantial evidence for the null hypothesis (Jeffreys, 1961).

\section{Results}

\section{Autobiographical memory}

We first tested the hypothesis that stimulation to the angular gyrus would reduce the number of internal details generated by participants during free recall of autobiographical memories (Fig. 2). To explore this issue, we used a repeated-measures ANOVA with three factors: region (left angular gyrus or vertex), recall type (free or cued), and detail type (internal or external). Our first question was whether angular gyrus cTBS affects free recall more than cued recall. There was a trend toward a main effect of region $\left(F_{(1,21)}=4.085, p=0.056, \eta_{\mathrm{p}}^{2}=0.163\right)$ and a significant effect of recall type $\left(F_{(1,21)}=99.394, p<0.001, \eta_{\mathrm{p}}^{2}=\right.$ $0.826)$. Critically, there was a significant interaction between region and recall type $\left(F_{(1,21)}=6.091, p=0.022, \eta_{\mathrm{p}}^{2}=0.225\right)$, which was driven by significantly fewer details reported during free recall after cTBS to the left angular gyrus compared with vertex stimulation $\left(t_{(21)}=3.199, p=0.004, d=0.682\right)$. No such reduction was observed during cued recall $\left(t_{(21)}=0.561, p=\right.$ $0.581, d=0.120)$. To further explore this null result, we used BF paired $t$ tests, which revealed a BF of 3.889 in favor of the null hypothesis, indicating substantial evidence against a stimulation effect.

Our next question was whether angular gyrus cTBS affects the production of specific internal details associated with the memory of interest rather than external irrelevant details. There was a significant interaction between region and detail type $\left(F_{(1,21)}=\right.$ 5.764, $\left.p=0.026, \eta_{\mathrm{p}}^{2}=0.215\right)$. Paired $t$ tests confirmed that this effect was driven by fewer internal details reported after angular gyrus cTBS $\left(t_{(21)}=3.147, p=0.005, d=0.671\right)$, with no differences observed for the production of external details $\left(t_{(21)}=\right.$ $0.929, p=0.364, d=0.198$ ). To further explore this null result, BF paired $t$ tests revealed a BF of 3.05 in favor of the null model, indicating substantial evidence against a stimulation effect. These results indicate that angular gyrus cTBS affected the production of relevant details when participants freely recalled autobiographical memories. Examining the different types of details (event, place, time, perceptual, and emotional) using paired $t$ tests revealed that the reduction in internal details was driven 
A

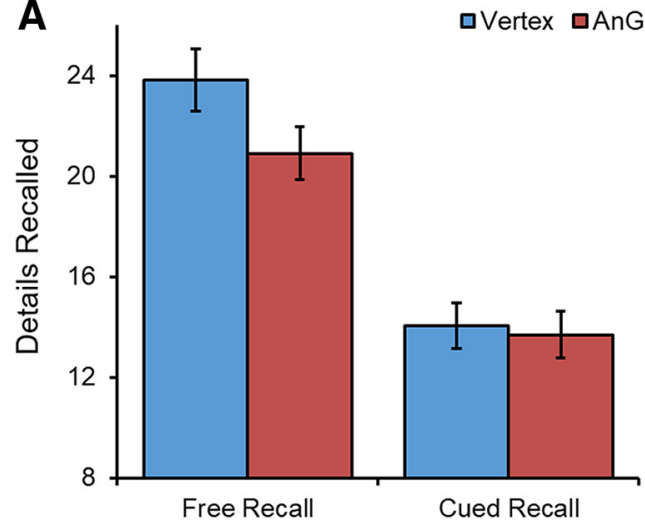

B

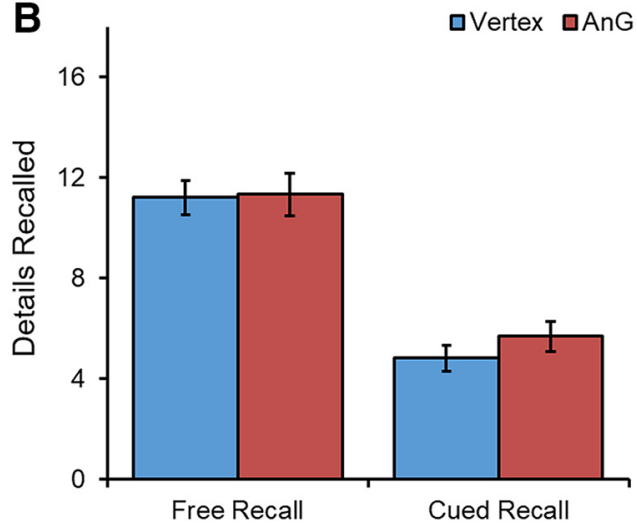

Figure 2. Mean number of $(\boldsymbol{A})$ internal details and $(\boldsymbol{B})$ external details produced by participants during free and cued autobiographical memory recall for vertex and left angular gyrus (AnG) stimulation. Significantly fewer internal details were produced after cTBS to the left angular gyrus during free recall.

Table 2. Freely recalled autobiographical memory internal detail types

\begin{tabular}{lllll}
\hline & & & \multicolumn{2}{l}{ Vertex versus AnG } \\
\cline { 5 - 6 } Detail type & Vertex [mean (SD)] & AnG [mean (SD)] & $t$ & $p$ \\
\hline Event & $10.1(3.9)$ & $8.2(3.5)$ & 3.539 & 0.002 \\
Place & $1.4(0.8)$ & $1.1(0.6)$ & 1.144 & 0.266 \\
Time & $1.3(0.8)$ & $1.2(0.7)$ & 0.648 & 0.524 \\
Perceptual & $8.5(3.9)$ & $7.9(4.8)$ & 0.742 & 0.466 \\
Emotion & $2.6(1.7)$ & $2.5(1.3)$ & 0.449 & 0.658 \\
\hline
\end{tabular}

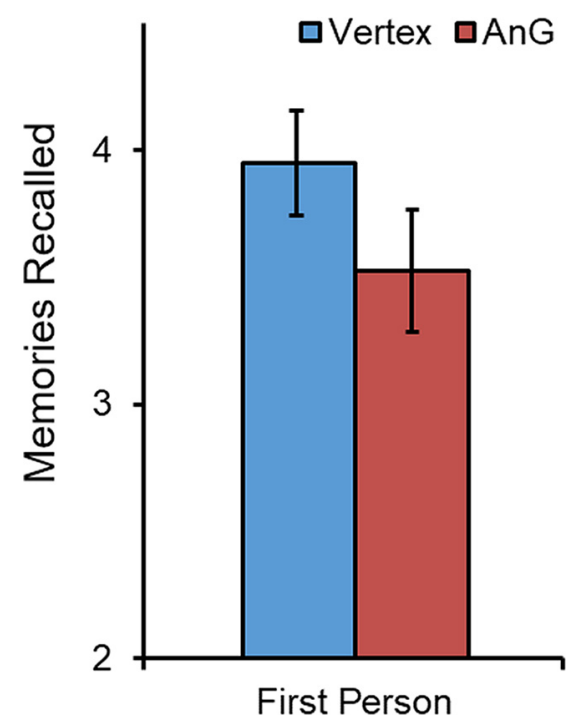

Figure 3. Mean number of autobiographical memories reported by participants as experienced from a first-person perspective following vertex and left angular gyrus (AnG) stimulation. Significantly fewer memories were experienced in the first-person after CTBS to the left angular gyrus.

specifically by fewer event details being reported $\left(t_{(21)}=3.539\right.$, $p=0.002$; Table 2).

\section{First-person versus third-person perspective}

Having obtained evidence that the left angular gyrus appears to be necessary for free recall of autobiographical memories, we next examined whether there was a difference in the perspective from which the participants experienced their memories (Fig. 3). Consistent with the hypothesis that angular gyrus is necessary for integrating memories within an egocentric framework, significantly fewer autobiographical episodes were reported as being

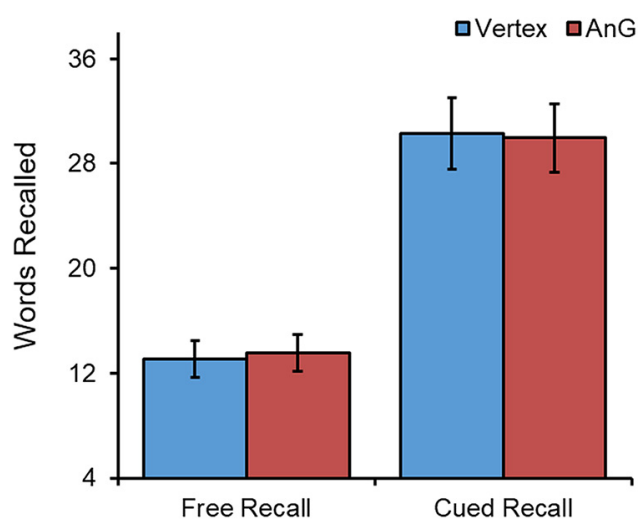

Figure 4. Mean number of recollected words during free and cued word-pair memory after vertex and left angular gyrus (AnG) stimulation. No significant difference in performance was observed for either type of recall.

experienced from a first-person perspective after angular gyrus cTBS compared with vertex stimulation $\left(t_{(18)}=2.191, p=0.042\right.$, $d=0.503)$.

\section{Word-pair memory}

We then examined the specificity of the observed reduction in free recall of autobiographical memories by testing whether cTBS stimulation affected recall of word pairs similarly (Fig. 4). A repeated-measures ANOVA with two factors: region (left angular gyrus or vertex) and recall type (free or cued), revealed no main effect of region $\left(F_{(1,21)}=0.008, p=0.932, \eta_{\mathrm{p}}^{2}=0.000\right)$, a significant effect of recall type $\left(F_{(1,21)}=75.743, p<0.001, \eta_{\mathrm{p}}^{2}=0.783\right)$, and no interaction between region and recall type $\left(F_{(1,21)}=\right.$ 0.462, $\left.p=0.504, \eta_{\mathrm{p}}^{2}=0.022\right)$. Consistent with these results, paired $t$ tests confirmed no significant differences between stimulation conditions during free recall $\left(t_{(21)}=0.468, p=0.645, d=\right.$ $0.100)$ and cued recall $\left(t_{(21)}=0.238, p=0.814, d=0.051\right) . \mathrm{BF}$ paired $t$ tests revealed a BF of 4.06 for free recall and 4.37 for cued recall in favor of the null model, providing substantial evidence for the null hypothesis of no stimulation effect. These results support previous findings that angular gyrus function is not necessary for recall of word pairs.

\section{Discussion}

The present experiment sought to determine the contribution made by angular gyrus to episodic memory by contrasting the predictions of two theories: (1) that it has a role in the capturing 
of attention by retrieved information, or (2) that its function is to enable the subjective experience that is associated with remembering. cTBS targeting angular gyrus compared with a vertex control site resulted in a selective reduction in the free recall, but not cued recall, of autobiographical memories, whereas free and cued recall of word-pair memories was unaffected. Additionally, angular gyrus cTBS led participants to report fewer autobiographical episodes as being experienced from a first-person perspective. These findings are consistent with the subjective experience account but less readily explained by the alternative attention-tomemory hypothesis, as is discussed below.

The observation that parietal lobe dysfunction was associated with disrupted autobiographical recall echoes the findings of several previous neuropsychology and neurostimulation studies (Berryhill et al., 2007, 2010; Davidson et al., 2008; Thakral et al., 2017). In particular, the significant reduction observed in the present data affecting free, but not cued, autobiographical recall is a direct replication of the result reported by Berryhill et al. (2007) in 2 patients with bilateral parietal lobe lesions. The present study followed the methodology for eliciting and scoring autobiographical memories used by Berryhill et al. (2007) closely and, like them, observed that parietal dysfunction was associated with selective impairment in the free recall of autobiographical events from participants' personal pasts, despite recall being unaffected when participants were cued by specific questions about the events. In the present data, the impairment in free autobiographical recall following angular gyrus cTBS was driven specifically by reduced production of "internal" details that were directly related to the probed event, rather than of "external" details that were irrelevant to the memory of interest. Berryhill et al., (2007) interpreted their results as consistent with a deficit in the bottom-up capturing of attention by salient information retrieved from episodic memory, although alternative accounts of parietal contributions to episodic memory retrieval have been proposed, such as sensitivity to the accumulation of mnemonic evidence (Wagner et al., 2005). However, a further feature of the present autobiographical recall data is difficult to accommodate within such accounts. Following angular gyrus cTBS, participants did not just freely recall fewer autobiographical event details but additionally reported fewer of their autobiographical memories to have been experienced from a first-person perspective. It is not clear how such a difference in the egocentric spatial perspective in which participants envisioned events from their personal pasts could be explained by a deficit in bottom-up attention, or other alternative accounts.

Further evidence against the attentional account comes from the observation that, although angular gyrus cTBS led to a significant reduction in free recall of autobiographical memories compared with stimulation of the vertex control site, it had no effect on free recall of word-pair memories. Support for the null hypothesis requires more than observation of a nonsignificant difference. Accordingly, BF analysis confirmed that the data provide substantial evidence against the prediction that because free recall relies more than cued recall on memories capturing attention spontaneously (Craik et al., 1996; Cabeza et al., 2008), angular gyrus disruption should produce a selective deficit in free recall of word pairs. Numerous previous studies have demonstrated that attentional manipulations impact free recall of words or word pairs to a greater degree than cued recall (e.g., Craik and McDowd, 1987; Craik et al., 1996). The observation in the present data that participants produced significantly fewer word-pair responses during free than cued recall, regardless of stimulation condition, is consistent with the more attentionally demanding nature of free recall in this task. Given that finding, the substantial evidence against an effect of angular gyrus cTBS on word-pair free recall is notable.

The observed results for word-pair recall replicate the previous neurostimulation findings reported by Yazar et al. (2014), who used a very similar task and cTBS protocol, and also observed that free and cued recall was unaffected by stimulation of angular gyrus compared with the vertex. Furthermore, the results are consistent with a previous neuropsychological study, which found that patients with parietal lobe lesions were unimpaired at recall of word-definition pairings (Davidson et al., 2008), but not with another study, which tested cued recall of word pairs in patients soon after they suffered posterior cortical strokes and identified performance deficits to be associated with damage affecting the angular gyrus (Ben-Zvi et al., 2015). Ben-Zvi et al. (2015) speculated that the findings of intact recall performance of Davidson et al. (2008) might be attributable to compensatory brain plasticity and reorganization due to testing taking place several years after damage occurred, as in many neuropsychological studies. Such an explanation would not seem sufficient to account for observations of unimpaired word-pair recall following angular gyrus cTBS in the present data and the results reported by Yazar et al. (2014), however. Whereas most studies of the parietal lobe and memory (the present experiment included) have focused on retrieval processes, it may be that the patient impairment reported by Ben-Zvi et al. (2015) could have arisen when the patients encoded the word pairs, an issue that future cTBS experiments might address. One other possible explanation, that a lack of observed difference could be attributable to insufficient power in the present experiment, is inconsistent with the results of the Bayesian analysis, which indicated that the data provided substantial evidence for null effects, rather than simply being insufficiently sensitive to detect true differences, and with the finding that power was sufficient to reveal a significant impairment in the free recall of autobiographical memories.

The present results add to a growing number of other findings that implicate the angular gyrus in processes that contribute to the subjective experience of remembering (Moscovitch et al., 2016). Subjective experiences associated with memory retrieval are complex and difficult to disentangle, which may be why the brain mechanisms underlying them have traditionally received less attention than more objective aspects of retrieval. Recent work has attempted to understand such experiential components of remembering in terms of their constituent cognitive processes, building on Tulving's (1983) seminal characterizations of "autonoetic" awareness, and to explore the extent to which predicted dissociations arise at behavioral and neural levels. Complementing findings such as those reported in the present experiment that parietal lobe dysfunction impairs participants' free recall of autobiographical events (Berryhill et al., 2007, 2010; Davidson et al., 2008; Thakral et al., 2017), performance deficits on other subjective measures of memory have also been reported. For example, neuropsychological and neurostimulation studies have observed reduced confidence in participants' accurate responses on source (Simons et al., 2010; Yazar et al., 2014) and associative (Berryhill et al., 2009) memory tasks, and that participants produce fewer "remember" responses on remember/know tasks (Davidson et al., 2008; Drowos et al., 2010). Angular gyrus disruption also leads to reduced performance on recollection tasks that require the multimodal integration of event features (Yazar et al., 2017) and on spatial navigation tasks that involve the sequencing of route landmarks from an egocentric perspective (Ciaramelli et al., 2010b). Consistent with this latter finding, angular gyrus 
cTBS in the present experiment resulted in fewer autobiographical memories being experienced from an egocentric perspective as opposed to an outside vantage point. Together, the existing data converge on the conclusion that angular gyrus might be the part of the network of brain regions involved in recollection that is specifically responsible for the subjective first-person "reliving" of personal events in all their multimodal glory that is such a defining feature of episodic memory (Tulving, 1983).

In conclusion, we found that cTBS targeting angular gyrus compared with a vertex control site was associated with selectively reduced free recall of autobiographical memories, but not of word-pair memories. Furthermore, angular gyrus cTBS resulted in fewer autobiographical events being experienced from a first-person perspective. These data build on a growing number of previous findings indicating a role for angular gyrus in producing the subjective experience of remembering.

\section{References}

Ben-Zvi S, Soroker N, Levy DA (2015) Parietal lesion effects on cued recall following pair associate learning. Neuropsychologia 73:176-194. CrossRef Medline

Berryhill ME, Phuong L, Picasso L, Cabeza R, Olson IR (2007) Parietal lobe and episodic memory: bilateral damage causes impaired free recall of autobiographical memory. J Neurosci 27:14415-14423. CrossRef Medline

Berryhill ME, Drowos DB, Olson IR (2009) Bilateral parietal cortex damage does not impair associative memory for paired stimuli. Cogn Neuropsychol 26:606-619. CrossRef Medline

Berryhill ME, Picasso L, Arnold R, Drowos D, Olson IR (2010) Similarities and differences between parietal and frontal patients in autobiographical and constructed experience tasks. Neuropsychologia 48:1385-1393. CrossRef Medline

Cabeza R, Ciaramelli E, Olson IR, Moscovitch M (2008) The parietal cortex and episodic memory: an attentional account. Nat Rev Neurosci 9:613625. CrossRef Medline

Ciaramelli E, Grady C, Levine B, Ween J, Moscovitch M (2010a) Top-down and bottom-up attention to memory are dissociated in posterior parietal cortex: neuroimaging and neuropsychological evidence. J Neurosci 30: 4943-4956. CrossRef Medline

Ciaramelli E, Rosenbaum RS, Solcz S, Levine B, Moscovitch M (2010b) Mental space travel: damage to posterior parietal cortex prevents egocentric navigation and reexperiencing of remote spatial memories. J Exp Psychol Learn Mem Cogn 36:619-634. CrossRef Medline

Craik FI, McDowd JM (1987) Age differences in recall and recognition. J Exp Psychol Learn Mem Cogn 13:474-479. CrossRef

Craik FI, Govoni R, Naveh-Benjamin M, Anderson ND (1996) The effects of divided attention on encoding and retrieval processes in human memory. J Exp Psychol Gen 125:159-180. CrossRef Medline
Davidson PS, Anaki D, Ciaramelli E, Cohn M, Kim AS, Murphy KJ, Troyer AK, Moscovitch M, Levine B (2008) Does lateral parietal cortex support episodic memory? Evidence from focal lesion patients. Neuropsychologia 46:1743-1755. CrossRef Medline

Dienes Z (2014) Using Bayes to get the most out of non-significant results. Front Psychol 5:781. CrossRef Medline

Drowos DB, Berryhill M, André JM, Olson IR (2010) True memory, false memory, and subjective recollection deficits after focal parietal lobe lesions. Neuropsychology 24:465-475. CrossRef Medline

Huang YZ, Edwards MJ, Rounis E, Bhatia KP, Rothwell JC (2005) Theta burst stimulation of the human motor cortex. Neuron 45:201-206. CrossRef Medline

Jeffreys H (1961) The theory of probability, Ed 3. Oxford: Oxford UP.

Levine B, Svoboda E, Hay JF, Winocur G, Moscovitch M (2002) Aging and autobiographical memory: dissociating episodic from semantic retrieval. Psychol Aging 17:677-689. CrossRef Medline

Moscovitch M, Cabeza R, Winocur G, Nadel L (2016) Episodic memory and beyond: the hippocampus and neocortex in transformation. Annu Rev Psychol 67:105-134. CrossRef Medline

Okamoto M, Dan H, Sakamoto K, Takeo K, Shimizu K, Kohno S, Oda I, Isobe S, Suzuki T, Kohyama K, Dan I (2004) Three-dimensional probabilistic anatomical cranio-cerebral correlation via the international 10-20 system oriented for transcranial functional brain mapping. Neuroimage 21: 99-111. CrossRef Medline

Rosenbaum RS, McKinnon MC, Levine B, Moscovitch M (2004) Visual imagery deficits, impaired strategic retrieval, or memory loss: disentangling the nature of an amnesic person's autobiographical memory deficit. Neuropsychologia 42:1619-1635. CrossRef Medline

Simons JS, Peers PV, Hwang DY, Ally BA, Fletcher PC, Budson AE (2008) Is the parietal lobe necessary for recollection in humans? Neuropsychologia 46:1185-1191. CrossRef Medline

Simons JS, Peers PV, Mazuz YS, Berryhill ME, Olson IR (2010) Dissociation between memory accuracy and memory confidence following bilateral parietal lesions. Cereb Cortex 20:479-485. CrossRef Medline

Thakral PP, Madore KP, Schacter DL (2017) A role for the left angular gyrus in episodic simulation and memory. J Neurosci 37:8142-8149. CrossRef Medline

Tulving E (1983) Elements of episodic memory. Oxford: Clarendon.

Vilberg KL, Rugg MD (2008) Memory retrieval and the parietal cortex: a review of evidence from a dual-process perspective. Neuropsychologia 46:1787-1799. CrossRef Medline

Wagner AD, Shannon BJ, Kahn I, Buckner RL (2005) Parietal lobe contributions to episodic memory retrieval. Trends Cogn Sci 9:445-453. CrossRef Medline

Yazar Y, Bergström ZM, Simons JS (2014) Continuous theta burst stimulation of angular gyrus reduces subjective recollection. PLoS One 9:e110414. CrossRef Medline

Yazar Y, Bergström ZM, Simons JS (2017) Reduced multimodal integration of memory features following continuous theta burst stimulation of angular gyrus. Brain Stim 10:624-629. CrossRef Medline 